\title{
Solid/Powder Clad Ti-6Al-4V Alloy with Low Young's Modulus and High Toughness Fabricated by Electron Beam Melting
}

\author{
Naoko Ikeo ${ }^{1, * 1}$, Takuya Ishimoto ${ }^{1}$, Natsumi Hiramoto ${ }^{1, * 2}$, Hidetsugu Fukuda ${ }^{2}$, \\ Hiroyuki Ogisu ${ }^{1, * 2}$, Yutaro Araki ${ }^{1, * 2}$ and Takayoshi Nakano ${ }^{1, * 3}$ \\ ${ }^{1}$ Division of Materials and Manufacturing Science, Graduate School of Engineering, Osaka University, Suita 565-0871, Japan \\ ${ }^{2}$ Nakashima Medical Co., Ltd, Okayama 709-0625, Japan
}

\begin{abstract}
A clad structure that consists of solid and powder parts was created from powdered Ti-6Al-4V utilizing electron beam melting (EBM) technique by a single process. The input energy density required to melt the raw powder material was controlled by changing the scan speed of the electron beam from 100 to $2000 \mathrm{~mm} / \mathrm{s}$. The finished products showed several types of structures: a dense solid, a periodic layered (clad) structure made up of a solid part and an unmelted powder part, and almost full powder. The products with the clad structure showed a combination of low Young's modulus and high toughness as characterized by the presence of a stress plateau in the stress-strain curve. Both of these qualities are necessary for feasibility as implant materials used in orthopedic fields. We conclude that the products developed in this study could be useful as bone implants in terms of the mechanical similarity to bones, despite needing only an single EBM process for fabrication. [doi:10.2320/matertrans.M2015025]
\end{abstract}

(Received January 16, 2015; Accepted February 17, 2015; Published April 10, 2015)

Keywords: electron beam melting, clad structure, biomaterials, low Young's modulus, toughness

\section{Introduction}

Additive manufacturing methodology has attracted increased attention in medical and industrial fields because of its potential to create three-dimensional (3D) products with arbitrary structure and function. Electron beam melting (EBM), a powder bed fusion technique, is widely used to rapidly fabricate dense metallic products and porous or lattice structures with a variety of geometries and densities ${ }^{1-4)}$ from powdered raw materials. Figure 1 is a schematic illustration of the EBM fabrication process. In EBM, an electron beam is scanned based on $2 \mathrm{D}$ slice data converted from a $3 \mathrm{D}$ computer-aided design (CAD) drawing of the final form. By repeating the process of selectively melting and solidifying one layer of metallic powder, multiple layers are stacked up to produce a $3 \mathrm{D}$ structure. Based on its ability to create an arbitrary structure, EBM technology enables to realize products with optimal integrated mechanical properties such as Young's modulus, yield stress, toughness, etc., with a high degree of freedom, making this technology promising for the fabrication of highly functionalized products. For example, the Ti-6Al-4V lattice structure with graded porosity exhibits a stepwise stress-strain curve with multiple yield stresses. ${ }^{5)}$

Absorption or dissipation of mechanical energy plays an increasingly important role for the integrity of structural materials subjected to a dynamic load. ${ }^{6}$ Recently, novel hierarchically-structured composites that were developed from a single material using EBM were shown to possess low Young's modulus and tunable energy absorption capacity (toughness). ${ }^{7}$ This composite is comprised of two types of components: a solid framework and powder particles inside the pores that are necked by optimal heat treatments. The combination of the solid and powdered parts is effective in

\footnotetext{
${ }^{* 1}$ Present address: Department of Mechanical Engineering, Graduate School of Engineering, Kobe University, Kobe 657-8501, Japan

${ }^{*}$ Graduate Student, Osaka University

${ }^{* 3}$ Corresponding author, E-mail: nakano@mat.eng.osaka-u.ac.jp
}

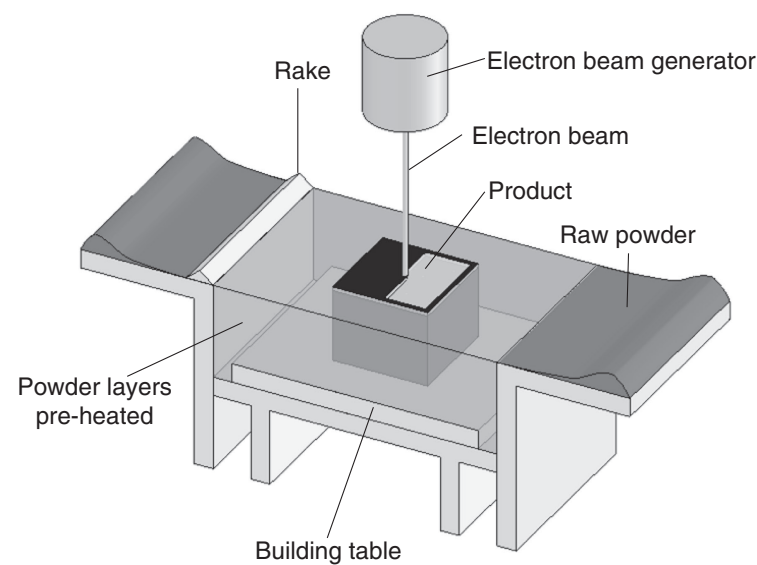

Fig. 1 Schematic image of the manufacturing process of EBM.

realizing a low Young's modulus and high toughness, ${ }^{7)}$ both of which are indispensable for use in orthopedic applications.

In the present study, we used EBM to fabricate a clad structure consisting of a melted solid part and a powder part using only a single process. We would like to stress that our procedure is done without any post processes such as heat treatment. By changing the scan speed of the electron beam, the thermal energy being input to melt the raw powder was optimally controlled to create clad products.

\section{Materials and Methods}

\subsection{Fabrication of specimens by EBM}

A gas-atomized Ti-6Al-4V ELI spherical powder (Arcam $\mathrm{AB}$, Sweden) with mean particle size of $78 \mu \mathrm{m}$ was used for $\mathrm{EBM}$ as a raw material. The composition of the powder fulfilled the standard specifications of ASTM F136.

A cuboid with dimensions of $5 \mathrm{~mm} \times 5 \mathrm{~mm} \times 10 \mathrm{~mm}$ was modeled using CAD software (SolidWorks, Dassault Systèmes SolidWorks Corp., France). The products were fabricated by EBM system according to the CAD drawing 
(Arcam12, Arcam AB, Sweden). The beam voltage, beam current, scan pitch, and lamination thickness were set to $60 \mathrm{kV}, 2 \mathrm{~mA}, 0.2 \mathrm{~mm}$, and $0.1 \mathrm{~mm}$, respectively. The scan speed of the electron beam was varied among 100, 500, 1000, 1500 , and $2000 \mathrm{~mm} / \mathrm{s}$. The slower electron beam scan results in a higher thermal energy input into the powder. Hereafter, the specimens fabricated with scan speeds of 100, 500, 1000, 1500 , and $2000 \mathrm{~mm} / \mathrm{s}$ are referred to as Specimen A, Specimen B, Specimen C, Specimen D, and Specimen E, respectively. Before fabrication, the raked powders were pre-heated to approximately $700^{\circ} \mathrm{C}$ by rapid electron beam scanning.

\subsection{Analysis of the fabricated specimens}

Apparent densities of the final products were calculated from the weights, apparent volumes, and the theoretical density of Ti-6Al-4V $\left(4.43 \mathrm{~g} / \mathrm{cm}^{3}\right)$. The lateral surfaces of specimens were observed by field emission scanning electron microscopy (FE-SEM; JSM6500, JEOL, Japan).

Uniaxial compression tests (AG-X, Shimadzu, Japan) were carried out at room temperature with an initial strain rate of $1.67 \times 10^{-4} \mathrm{~s}^{-1}$. Strain gages (KFG-4N-120-C1, PCD-330B, Kyowa electronic instruments, Japan) were used to detect strain during the compression tests for accurate evaluation of the Young's modulus. The loading direction was parallel to the building direction of the specimens.

Three specimens in each group were used for all quantitative analyses, and the results are presented as means \pm standard deviations (SD). Statistical comparisons between two means were performed using a two-tailed unpaired Student's t-test. Comparisons of means among groups of more than two were performed using one-way analysis of variance (ANOVA) and following a post-hoc multiple comparison test (Scheffe's test). A value of $P<0.05$ was considered statistically significant. SPSS version 14.0J software (SPSS Japan Inc., Japan) for Microsoft Windows was used for all statistical analyses.

\section{Results and Discussion}

\subsection{Analyses of the specimen's structure fabricated through various scan speeds of the electron beam}

Raw powder particles are selectively melted according to the energy density of the electron beam radiated on them. In general, the energy density of the electron beam per unit volume (ED) is given by the following equation: ${ }^{8)}$

$$
\mathrm{ED}=P /(h \times v \times t),
$$

where $P$ is the electron beam power, $h$ is the scan pitch, $v$ is the scan speed, and $t$ is the lamination thickness. Figure 2 shows the variation of the energy density as a function of the electron beam scan speed. The energy density input to the powder particles decreases as electron beam scan speed increases.

Figure 3 shows FE-SEM images of the lateral surface of the products fabricated by EBM. The arrows in these images indicate the building direction. As shown in Fig. 3(a), Specimen A was fabricated by a slow electron beam scan $(100 \mathrm{~mm} / \mathrm{s})$. Specimen A has thick and dense solidified layers that are tightly connected to one another. The powder

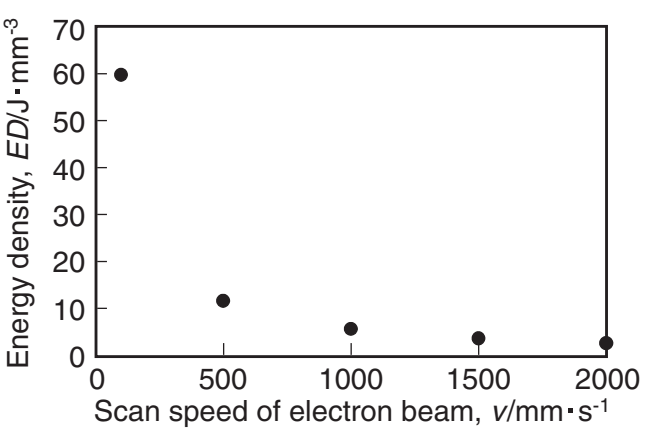

Fig. 2 The relationship between input energy density from the electron beam and the electron beam scan speed.

particles fed on the building table were fully melted by the slow electron beam irradiation. Unmelted powder particles are also present at the outer surface because the melt pool formed by electron beam irradiation trapped adjacent powder particles. Other powder particles untrapped by the melt pool were removed after fabrication. When the scan speed of the electron beam was 500 and $1000 \mathrm{~mm} / \mathrm{s}$, there are periodic layered solid parts perpendicular to the building direction as shown in Fig. 3(b) and (c). The solid plates are thicker in Specimen B than in Specimen C because of difference in the thermal energy input to the powder particles. In the both specimens, an abundance of unmelted powder particles were trapped between the melted solid plates, forming a clad structure made up of melted solid and powder parts with a relatively regular stacking. The height of the pore spaces between the solid plates where the powder particles lie is less than the size of two particles. The pores function as an energy absorber ${ }^{7}$ and contribute to reduce the Young's moduli of the products. In contrast, under fast scanning of the electron beam (1500 and $2000 \mathrm{~mm} / \mathrm{s})$, powder particles were rarely melted because a fast electron beam scan speed causes a small heat input, resulting in a smaller size of the heated volume. $^{9)}$ In Specimen D, a few solidified parts are discontinuously found (Fig. 3(d)).

Figure 4 shows the variation of the relative density as a function of the scan speed of the electron beam. The relative density monotonically decreases with increasing scan speed. The relative density of Specimen E is similar to the packing density of the pre-heated powder, ${ }^{7)}$ indicating that the electron beam scan speed of $2000 \mathrm{~mm} / \mathrm{s}$ hardly affects the raw powder.

The scan speed of the electron beam in the EBM fabrication is one of the important process parameters ${ }^{10)}$ in addition to the beam current, beam size, and so on. Therefore, some reports focused on the effect of the electron beam scan speed on the microstructure, porosity (as a defect), and surface roughness. ${ }^{11,12)}$ However, to our knowledge, no reports were found where a clad structure developed through EBM by intentionally leaving powder particles for functionalizing the products.

\subsection{Mechanical properties of the specimens determined by compression test}

Figure 5 shows the stress-strain curves obtained by uniaxial compression tests parallel to the building direction. 
(a) Specimen A (100 mm/s)

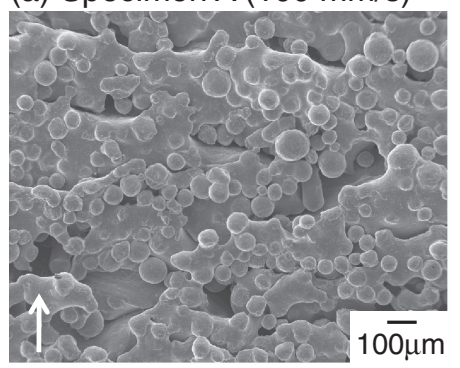

(d) Specimen D (1500 mm/s)

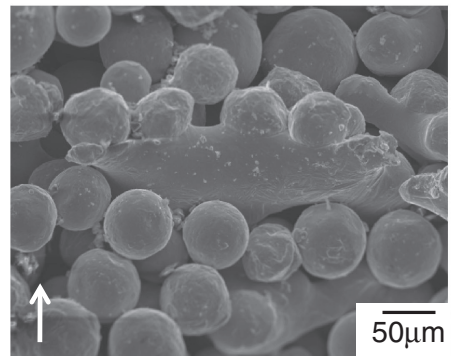

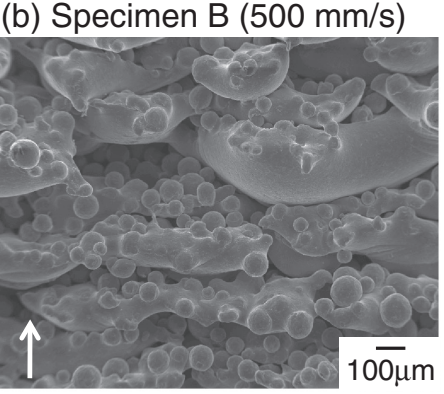

(e) Specimen E $(2000 \mathrm{~mm} / \mathrm{s})$

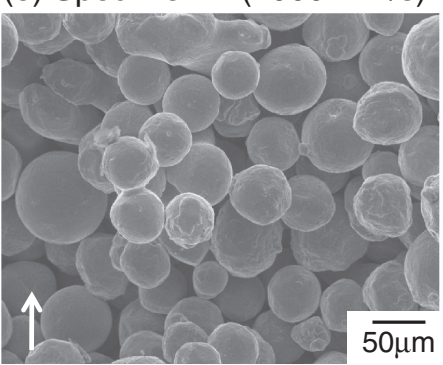

(c) Specimen C $(1000 \mathrm{~mm} / \mathrm{s})$

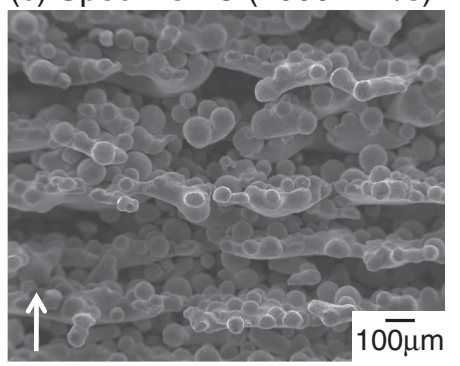

Fig. 3 SEM images of the lateral surface of specimens fabricated with various scan speed: (a) $100 \mathrm{~mm} / \mathrm{s}$, (b) $500 \mathrm{~mm} / \mathrm{s}$, (c) $1000 \mathrm{~mm} / \mathrm{s}$,

(d) $1500 \mathrm{~mm} / \mathrm{s}$, and (e) $2000 \mathrm{~mm} / \mathrm{s}$. Arrows indicate the building direction.

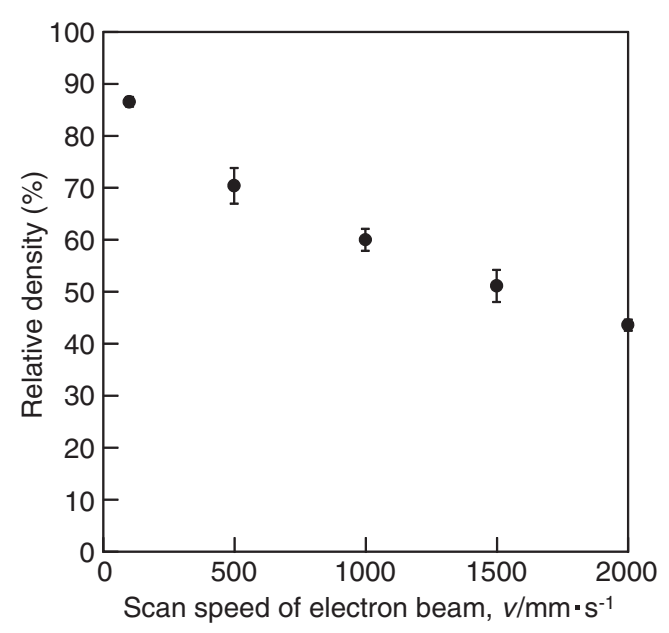

Fig. 4 Variation in relative density as a function of the electron beam scan speed. $P<0.05$ by one-way ANOVA.

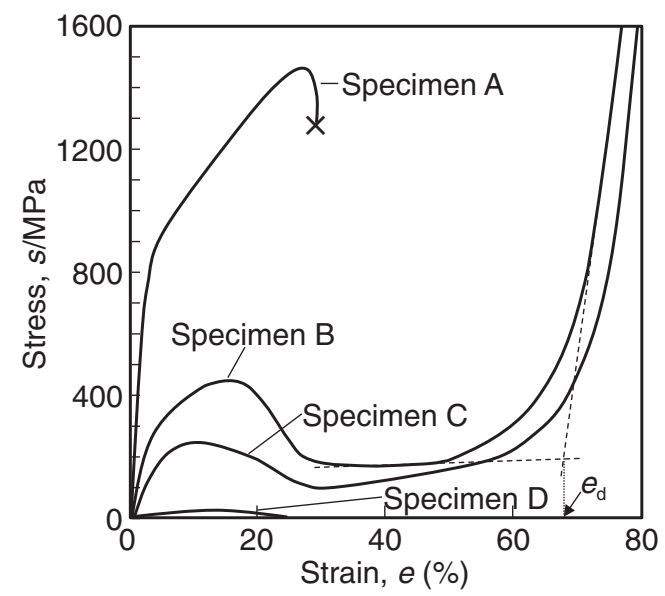

Fig. 5 Stress-strain curves of the specimens fabricated with various scan speeds. The curve for Specimen E is not shown because it had almost no stress sustainment.
Table 1 Mechanical properties of specimens as determined from the stressstrain curves.

\begin{tabular}{ccccc}
\hline & Specimen A & Specimen B & Specimen C & $\begin{array}{c}\text { Statistical } \\
\text { significance }\end{array}$ \\
\hline $\begin{array}{c}\text { Young's modulus } \\
E / \mathrm{GPa}\end{array}$ & $75.7 \pm 4.3$ & $38.9 \pm 1.0$ & $31.3 \pm 2.3$ & $*_{1}$ \\
\hline $\begin{array}{c}\text { Yield stress } \\
s_{\mathrm{y}} / \mathrm{MPa}\end{array}$ & $716 \pm 13$ & $216 \pm 19$ & $134 \pm 8$ & $*_{1}$ \\
\hline $\begin{array}{c}\text { Plateau stress } \\
s_{\mathrm{p}} / \mathrm{MPa}\end{array}$ & - & $328 \pm 26$ & $193 \pm 58$ & $*_{2}$ \\
\hline $\begin{array}{c}\text { Toughness } \\
u / \mathrm{MJ}^{-3}\end{array}$ & - & $220 \pm 21$ & $154 \pm 16$ & $*_{2}$ \\
\hline
\end{tabular}

${ }^{* 1} P<0.05$ between Specimen A and Specimen B, Specimen B and Specimen C, and Specimen C and Specimen A, by Scheffe's test.

${ }^{* 2} P<0.05$ between Specimen B and Specimen C by Student's t-test.

Because Specimen E did not support stress, the curve for Specimen $\mathrm{E}$ is not present. The deformation behavior can be classified into three groups: (i) Specimen A, (ii) Specimen B and Specimen C, and (iii) Specimen D. Specimen A was fabricated by a slow electron beam scan speed of $100 \mathrm{~mm} / \mathrm{s}$ and shows highest density of about $87 \%$ exhibited elastic deformation followed by work hardening, and finally underwent fracture immediately after reaching maximum stress and it does not show a plateau region. Meanwhile, Specimen B and Specimen $\mathrm{C}$ show a plateau region during which deformation progresses at nearly constant stress. After a certain amount of densification, stress rapidly increased. Specimen D and Specimen E were fabricated with a fast electron beam scan speed and barely supported stress.

Some important mechanical outputs for Specimen A, Specimen B, and Specimen C were extracted from the stress-strain curves and are summarized in Table 1. Young's modulus and yield stress decreased as a function of electron 
beam scan speed and resultant relative density. Plateau stress and toughness were calculated only for Specimen B and Specimen C, both of which showed a plateau region (Fig. 5). Plateau stress was defined as the average value of the stress within the plateau region. According to JIS H 7902, plateau stress is defined as the average stress within the strain range of $20 \%$ to $30 \%,{ }^{13)}$ however, the plateau regions appeared in at much higher strains in the present study. The absorption energy per unit volume (toughness), $W$, is evaluated by integrating the area under the stress-strain curve:

$$
W=\int_{0}^{e_{\mathrm{d}}} s(e) d e,
$$

where $e_{\mathrm{d}}$ is the densification strain, taken as the intersection of the plateau tangent and the densification tangent on the stress-strain curve, as illustrated in Fig. 5.

Specimen B and Specimen C possesses clad structures and showed clear plateau regions. In these plateau regions, pores in the powdered part between solid plates experience collapses and densification through which energy from an external load is dissipated, ${ }^{14-16)}$ leading to high toughness. Here, the co-existence of the solid part and the powder part is considerably effective at making the products tough and energy absorptive, similar to the other reports. ${ }^{7)}$

As materials for orthopedic applications, a low Young's modulus is also required to prevent stress shielding. Stress shielding is defined as the removal of normal stress from the bone by insertion of an implant with high Young's modulus. The Young's moduli of cortical bones typically range from 10 to $30 \mathrm{GPa},{ }^{17)}$ while those of commercially used metallic materials in orthopedics are significantly higher. For example, the alloy Ti-6Al-4V shows $110 \mathrm{GPa}$. Removal of stress from the bone results in bone resorption, ${ }^{18)}$ degradation of bone quality ${ }^{19)}$ which is partly characterized by the preferential orientation of collagen and apatite, ${ }^{20-22)}$ and resultant reduced mechanical properties such as hardness, ${ }^{23)}$ strength, and toughness. ${ }^{24)}$ The products fabricated in the present study have low Young's moduli, less than $40 \mathrm{GPa}$, which is close to those of bones. At the same time, yield stress is around $200 \mathrm{MPa}$, which is also close to that of bones, and it can be further modified by changing the scan speed of electron beam. Therefore, the products with clad structure should be promising for use in orthopedic applications. They may serve as bone replacement materials to reduce the negative effects of stress shielding onto bones.

\section{Conclusions}

The present study developed the solid/powder clad structures by a single process of EBM, and their structural and mechanical properties were analyzed. The key findings are as follows:

(1) Several types of structures were achieved through EBM by controlling the electron beam scan speed to optimize the energy density for melting raw powder. Products fabricated with a certain range of scan speeds demonstrated a clad structure consisting of periodic layers of a melted solid part and a powder part.

(2) Mechanical properties derived from compression tests varied as a function of the electron beam scan speed and the resultant structure of the products. The products with the clad structure mentioned above showed a low Young's modulus and high toughness, both of which are important for orthopedic usage. Therefore, the developed products here could be potent as bone implant materials or for use in the inner structure of implants.

\section{Acknowledgement}

This work was partly supported by the Grants-in-Aid for Scientific Research from the Japan Society for the Promotion of Science (JSPS) and the Funds for Integrated Promotion of Social System Reform and Research and Development, the Support Program to break the bottlenecks at R\&D System for accelerating the practical use of Health Research Outcome, from the Japan Science and Technology Agency (JST). T. Nakano thanks the Iketani Science and Technology Foundation for partially supporting this research.

\section{REFERENCES}

1) X. Y. Cheng, S. J. Li, L. E. Murr, Z. B. Zhang, Y. L. Hao, R. Yang, F. Medina and R. B. Wicker: J. Mech. Behav. Biomed. Mater. 16 (2012) $153-162$.

2) N. Ikeo, T. Ishimoto, A. Serizawa and T. Nakano: Metall. Mater. Trans. A 45 (2014) 4293-4301.

3) T. J. Horn, O. L. A. Harrysson, D. J. Marcellin-Little, H. A. West, B. D. X. Lascelles and R. Aman: Addit. Manufact. 1-4 (2014) 2-11.

4) F. A. List, R. R. Dehoff, L. E. Lowe and W. J. Sames: Mater. Sci. Eng. A 615 (2014) 191-197.

5) W. van Grunsven, E. Hernandez-Nava, G. C. Reilly and R. Goodall: Metals 4 (2014) 401-409.

6) L. Sun, R. F. Gibson, F. Gordaninejad and J. Suhr: Compos. Sci. Technol. 69 (2009) 2392-2409.

7) N. Ikeo, T. Ishimoto and T. Nakano: J. Alloys Compd. 639 (2015) 336340 .

8) M. F. Zaeh and M. Kahnert: Prod. Eng. Res. Devel. 3 (2009) 217-224.

9) M. Jamshidinia, F. Kong and R. Kovacevic: Mech. Eng. Res. (2013) Paper 4.

10) X. Gong, T. Anderson and K. Chou: Manufact. Rev. 1 (2014) Paper 2.

11) K. Puebla, L. E. Murr, S. M. Gaytan, E. Martinez, F. Medina and R. B. Wicker: Mater. Sci. Appl. 3 (2012) 259-264.

12) A. Safdar, H. Z. He, L. Y. Wei, A. Snis and L. E. Chavez de Paz: Rapid Prototyping J. 18 (2012) 401-408.

13) JIS H 7902: Method For Compressive Test Of Porous Metals (2008).

14) W. Niu, C. Bai, G. Qiu and Q. Wang: Mater. Sci. Eng. A 506 (2009) $148-151$.

15) Y. Chino and D. C. Dunand: Acta Mater. 56 (2008) 105-113.

16) Z. Esen and Ș. Bor: Mater. Sci. Eng. A 528 (2011) 3200-3209.

17) M. Niinomi: J. Mech. Behav. Biomed. Mater. 1 (2008) 30-42.

18) H. Weinans, D. R. Sumner, R. Igloria and R. N. Natarajan: J. Biomech. 33 (2000) 809-817.

19) Y. Noyama, T. Nakano, T. Ishimoto, T. Sakai and H. Yoshikawa: Bone 52 (2013) 659-667.

20) T. Nakano, K. Kaibara, Y. Tabata, N. Nagata, S. Enomoto, E. Marukawa and Y. Umakoshi: Bone 31 (2002) 479-487.

21) T. Nakano, K. Kaibara, T. Ishimoto, Y. Tabata and Y. Umakoshi: Bone 51 (2012) 741-747.

22) T. Ishimoto, T. Nakano, Y. Umakoshi, M. Yamamoto and Y. Tabata: J. Bone Miner. Res. 28 (2013) 1170-1179.

$23)$ G. X. Ni, W. W. Lu, P. K. Y. Chiu, Y. Wang, Z. Y. Li, Y. G. Zhang, B. $\mathrm{Xu}$, L. F. Deng and K. D. K. Luk: J. Orthop. Res. 25 (2007) 14081414.

24) P. Låftman, F. Sigurdsson and L. Strömberg: Acta Orthop. Scand. 51 (1980) 215-222. 